\title{
Deferring stent optimization in stent thrombosis: A novel approach for STEMI management - Insights from a case series
}

\author{
Giacomo Cioffi $^{1}$, Mehdi Madanchi ${ }^{1}$, Matthias Bossard ${ }^{1}$, and Florim Cuculi ${ }^{1}$ \\ ${ }^{1}$ Luzerner Kantonsspital
}

October 28, 2020

\begin{abstract}
Treatment of ST represents a challenge. The presence of large amounts of thrombus combined with stent optimization increase the risk of distal embolization. A two-step strategy of stent implantation and deferred stent optimization might be appropriated. We hereby present three clinical cases of ST successfully treated with a two-step approach.
\end{abstract}

\section{INTRODUCTION}

Nowadays, stent thrombosis (ST) represents a rare but still devastating outcome after percutaneous coronary intervention (PCI) with contemporary stent platforms. The majority of patients present with acute myocardial infarction (AMI), mostly ST-segment elevation myocardial infarction (STEMI), due to thrombotic occlusion of the previously implanted stent. [1,2]

Two issues may make the treatment of ST challenging. First, intravascular imaging (especially optical coherence tomography, OCT) is key to understand the mechanism of ST (differentiation between mechanical and pharmacological factors), but due to the presence of large amounts of thrombus image interpretation is often hampered. Second, in order to correct mechanical factors, particularly under-expansion, the use of appropriately sized balloons (often at high pressure) is necessary, which in turn increases the risk for distal embolization and flow deterioration, thus increasing myocardial infarction size.

In this context, we present three cases of STEMI patients with ST whom we treated with a novel approach aiming to mitigate the risk for distal embolization. Given the large thrombus burden in ST, we therefore followed a two-step strategy of stent implantation and deferred stent optimization using intravascular imaging.

\section{CASE PRESENTATION}

\section{Case 1}

A 48-year-old female presented with an inferior STEMI. She had undergone PCI and implantation of a drug eluting stent (DES) to the proximal right coronary artery (RCA) for an AMI five years earlier (Fig 1 A, B). The angiogram revealed a thrombotic occlusion (TIMI 0 flow) due to very late ST of the previously implanted DES. The angiogram indicated a large thrombus burden. A bolus of intracoronary eptifibatide was administered. After pre-dilatation using a $2.0 \mathrm{~mm}$ non-compliant (NC) balloon and thrombectomy (ASAP LP, Merit Medical, U.S.A.), we were not able to completely restore flow (TIMI 1) (Fig 1 C). The OCT investigation (Dragonfly, Abbott Vascular, Santa Clara, California) indicated presence of large amount of white and red thrombus and a previously implanted stent, which appeared under-expanded (Fig 2 A). Additionally, we observed excessive neointima formation. We consecutively implanted a 3.5x28 mm DES (Xience Sierra, Abbott Vascular, Santa Clara, CA) at nominal pressure (12 atm), which immediately established TIMI 3 flow and lead to complete ST-segment resolution (Fig 1 D). Stent-optimization was postponed, and the patient was 
treated with dual antiplatelet therapy and therapeutic dosage of dalteparine. Five days later, OCT-guided stent optimization was performed. Small amounts of residual thrombus were still present, and we observed under-expansion of the initial stent as well as under-expansion and malapposition of the newly implanted stent (Fig 2 B). A $4.0 \mathrm{~mm}$ (NC) balloon (Easy T NC, SIS Medical Switzerland, Frauenfeld Switzerland) was used (inflation pressure $25 \mathrm{~atm}$ ), which corrected stent under-expansion and improved stent apposition, as confirmed by angiography and OCT (Fig 1 E, F; Fig 2 C). Coronary flow was not impacted (final TIMI flow 3 ). Our patient was discharged after 6 days. She recovered well and her follow-up echocardiogram revealed an only mildly reduced left ventricular ejection fraction (LVEF $50 \%$ ).

\section{CASE 2}

A 57-year-old male, with a history of an anterior STEMI requiring stenting of the proximal left anterior descending (LAD) 2 years earlier, was admitted with a repeated anterior STEMI due to very late ST. Predilatation with $2.0 \mathrm{~mm}$ balloon at very low pressure $(6 \mathrm{~atm})$ and bailout thrombectomy (ASAP LP catheter, Merit Medical) due to large thrombus amount were performed. Additionally, intracoronary eptifibatide and adenosine were administered, but TIMI 3 flow was only established after placement of a 3.0x18 mm DES (Xience Sierra, Abbott Vascular) at nominal pressure in the proximal LAD. No post-dilatation (PD) was performed. Four days later, OCT-guided PCI was performed and confirmed an under-expanded stent, but no residual thrombus. A $3.5 \mathrm{~mm}$ ultra-high NC balloon (OPN NC, SIS Medical, Frauenfeld Switzerland) at $35 \mathrm{~atm}$ was used to enhance stent expansion and an additional DES was implanted distally to correct a geographical miss. Ultimately, a $3.5 \mathrm{~mm}$ semi-compliant (SC) balloon was used to achieve optimal stent apposition, which was confirmed on OCT. The patient was discharged on day 5 . His left ventricular function had almost normalized at follow-up 17 months later (LVEF 52\%).

\section{CASE 3}

A 62-year-old male, with a history of a NSTEMI 7 years earlier requiring treatment of the proximal LAD using a DES, underwent primary angioplasty for anterior STEMI due to very late LAD ST. After pre-dilatation with a $2.0 \mathrm{~mm} \mathrm{SC}$ balloon and administration of intracoronary eptifibatide, a $3.5 \mathrm{~mm}$ DES (Xience Sierra, Abbott Vascular) was implanted at nominal pressure, which immediately re-established TIMI 3 flow. Four days later OCT guided stent optimization was performed using a $3.5 \mathrm{~mm}$ OPN NC balloon at 40 atm to correct under-expansion and $4.0 \mathrm{~mm} \mathrm{SC}$ balloon at $16 \mathrm{~atm}$ to optimize apposition. His follow-up remained uneventful and he recovered well. Of note, his latest LVEF was $55 \%$ with mild anterior hypokinesia.

\section{DISCUSSION}

We need to take into account that advances in AMI management, including potent antithrombotic drugs, rapid interventional reperfusion strategies and advancements in coronary stent designs have rendered ST a rare complication after DES implantation. The incidence of ST at 30 days after PCI is $<1 \%$, whereas late and very late ST rates are $0.5-1 \%$ and $0.2-2 \%$ per year, respectively.[3]

Established factors concurring to ST include: first, stent under-expansion and malapposition, which are especially common in calcified and tortuous vessels; second, stent placement in small vessels (vessel diameter $<2.5 \mathrm{~mm}$ ) and/or a long lesion may also be related to impaired and turbulent coronary flow; third, edge dissections compromising coronary flow; finally, hypercoagulability or inappropriate platelet inhibition (e.g. non-response to clopidogrel).[4] According to the timing of the event, the ST is labeled as early (within one month of initial placement), late (between 1 and 12 months), and very late (after 12 months).

In this report, we are focusing on ST due to stent under-expansion and describe how we addressed this issue in the three patients presented above.

Current guidelines recommend intravascular imaging to elucidate the mechanism of ST. Due to the higher spatial resolution, OCT might provide more detailed information about stent apposition, stent expansion and the presence of relevant edge dissections. But performing OCT imaging during acute STEMI is challenging due to the presence of large amounts of thrombus making visualization of relevant portions of the stent 
impossible. Additionally, vessel size is likely to be underestimated in the acute setting due to high levels of circulating vasoconstrictive hormones, namely catecholamines and vasopressin.

In acute ST with AMI, it is crucial to achieve TIMI 3 flow as soon as possible without going through a phase of flow-deterioration, which is usually the consequence of distal embolization of clot, microvascular spasm, thrombosis and friable atheromatous plaques.[5] The "burden" of thrombus in patients with STEMI undergoing primary PCI has been identified as a major determinant of outcomes, having been associated with reduced procedural success and worse early and late event-free survival.[6]

Moreover, it is crucial to correct mechanical factors (particularly stent under-expansion) in order to correct the cause of ST and prevent repeat target lesion failure. In this context, aggressive mechanical expansion is a known risk factor for distal embolization and microvascular injuries, especially for patients with AMI.[7]

\section{CONCLUSIONS}

Our case series suggests that applying a two-step approach (Central Illustration) in patients with STEMI due to ST is safe and effective. After mechanical recanalization with small balloons and/or manual thrombectomy, we advocate the implantation of an appropriately sized stent at nominal pressure strictly avoiding postdilatation. In the three cases presented, stent implantation immediately restored TIMI 3 flow and the procedure could be finished quickly. We did not observe any flow deterioration (slow-flow or no-reflow) secondary to distal embolization. When bringing the patients back for stent optimization, usually 3-5 days later, we used OCT to elaborate the mechanism of ST, assess vessel size and check for geographic miss. Use of aggressive balloon dilatation was well tolerated at this point in time and did not cause slow-flow or even no-reflow. Whether this strategy would be a valid approach in larger cohort of patients with STEMI remains to be seen. However, we believe that provisional stent implantation to push thrombus aside and re-establish flow (and optimize 3-5 days later) in ST might reflect a promising and safe approach for patients with STEMI presentation and large thrombus burden.

\section{Take Home Messages:}

- ST is a feared acute complication of stent implantation usually resulting in acute coronary syndrome

- Under-expanded or malapposed stents, edge dissections and hypercoagulability states are the main factors concurring in ST

- Deferred PD or stent optimization might be a possibile strategy to avoid no-reflow phenomenon, distal embolization or MVO during STEMI settings

\section{Authors Contribution}

Cioffi GM and Madanchi M: drafting, critical reviewing, final revision, image and data analisys.

Cioffi GM and Cuculi F: Central Illustration and figures

Cuculi F and Bossard M: conceptualization, critical reviewing and final revision

\section{Acknowledgement}

No external financial funding or material was provided for this work.

The authors received no specific funding for this work

\section{References}

1. Longobardo, L., et al., OCT-guided Percutaneous Coronary Intervention in Bifurcation Lesions. Interv Cardiol, 2019.14 (1): p. 5-9.

2. Lee, S.N., et al., The Glasgow prognostic score as a significant predictor of clinical outcomes in patients with acute coronary syndrome. J Cardiol, 2019. 74 (2): p. 130-135.

3. Gori, T., et al., Predictors of stent thrombosis and their implications for clinical practice. Nat Rev Cardiol, 2019.16 (4): p. 243-256. 
4. Ge, J., H. Yu, and J. Li, Acute Coronary Stent Thrombosis in Modern Era: Etiology, Treatment, and Prognosis. Cardiology, 2017.137 (4): p. 246-255.

5. Carrick, D., et al., A randomized trial of deferred stenting versus immediate stenting to prevent no- or slow-reflow in acute ST-segment elevation myocardial infarction (DEFER-STEMI). J Am Coll Cardiol, 2014. 63 (20): p. 2088-2098.

6. Costa, R.A., et al., Impact of thrombus burden on outcomes after standard versus mesh-covered stents in acute myocardial infarction (from the MGuard for acute ST elevation reperfusion trial). Am J Cardiol, 2015. 115 (2): p. 161-6.

7. Gao, P., et al., Application of post-dilation in ST-segment elevation myocardial infarct

patient undergoing primary percutaneous coronary intervention. Int $J$

Clin Exp Med. 2018;11(11):12657-12663.

\section{Figure legends}

Figure 1 - Angiography A, B - PCI and DES to the proximal right coronary artery (RCA) for an AMI five years earlier. C - Pre-dilatation and thrombectomy not able to completely restore flow (TIMI 1). D Implantation of a DES at nominal pressure immediately restored TIMI 3 flow. E, F - Post-dilatation with a NC balloon corrected stent under-expansion and improved stent apposition.

Figure 2 - OCT A - Presence of large amount of white and red thrombus, excessive neointima formation and a previously implanted and under-expanded stent. B - Five days later, OCT-guided stent optimization showed residual thrombus, under-expansion and malapposition of the newly implanted stent. C - Aggressive post-dilatation with $\mathrm{NC}$ balloon corrected stent under-expansion and improved stent apposition.

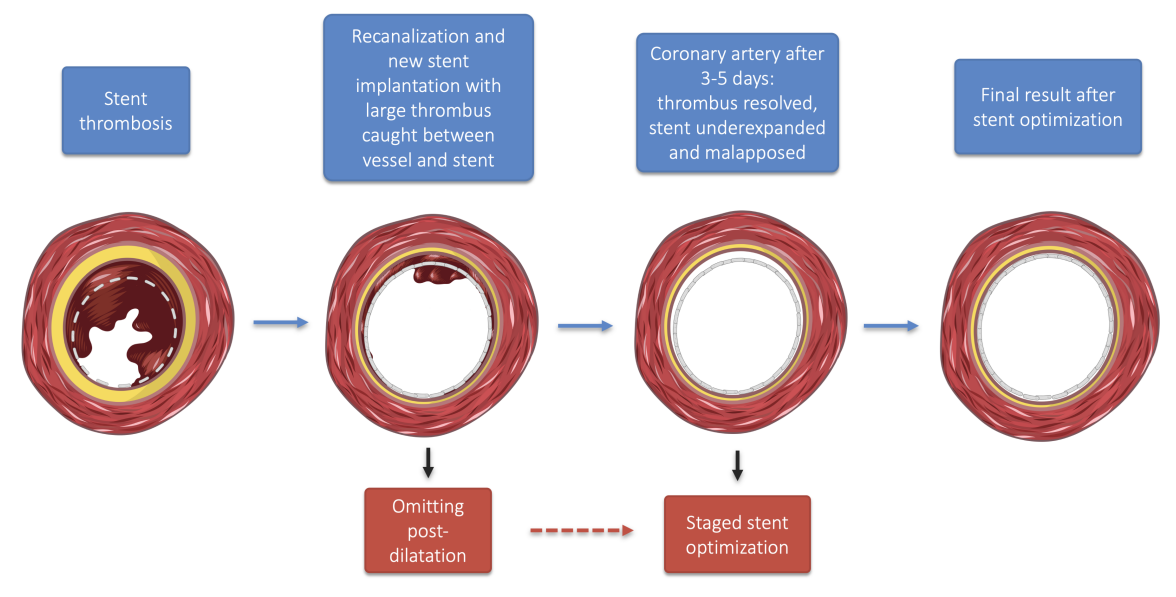


A
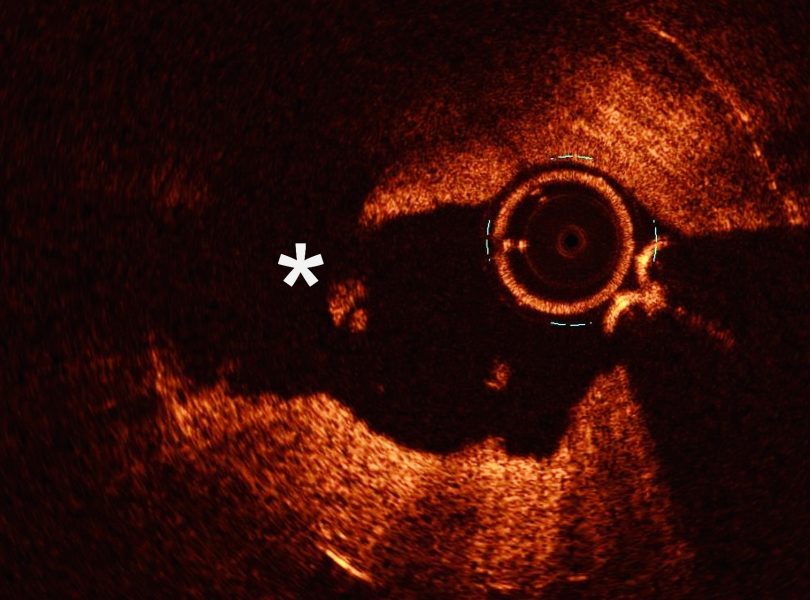

B
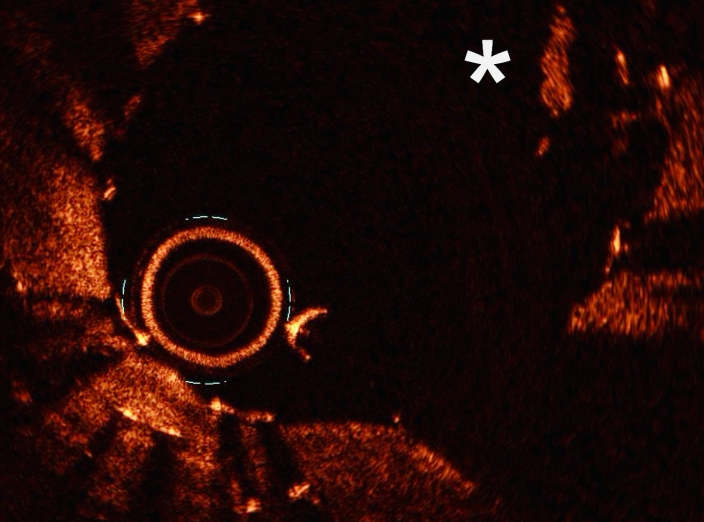

C

30 .

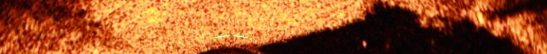
ค 


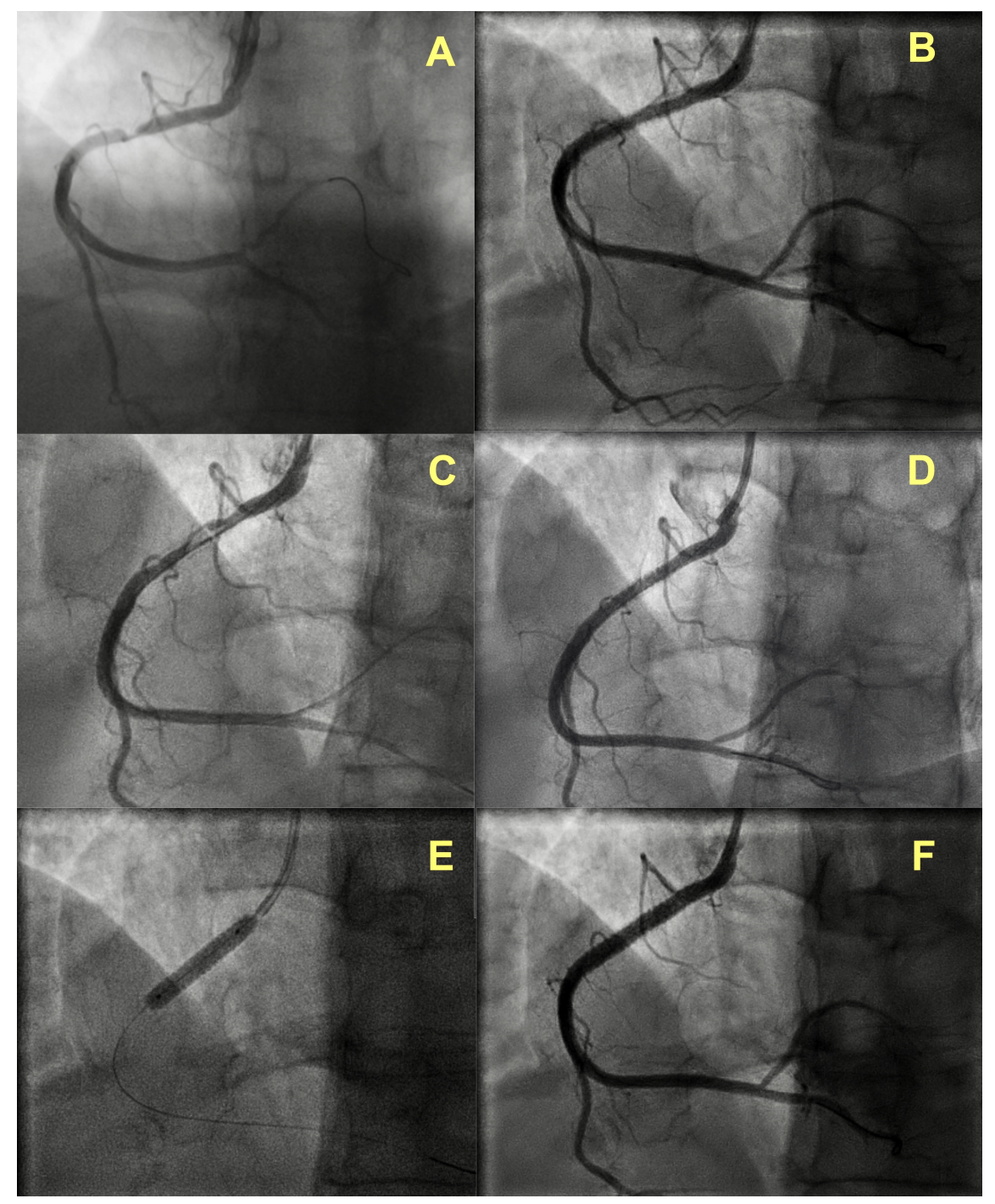

